\title{
Dynamics of hard colloidal cuboids in nematic liquid crystals
}

\author{
Alejandro Cuetos $\oplus^{1, *}$ and Alessandro Patti $\odot^{2, \dagger}$ \\ ${ }^{1}$ Department of Physical, Chemical and Natural Systems, Pablo de Olavide University, 41013 Sevilla, Spain \\ ${ }^{2}$ Department of Chemical Engineering and Analytical Science, The University of Manchester, Manchester M13 9PL, United Kingdom
}

(Received 17 February 2020; accepted 18 March 2020; published 18 May 2020; corrected 26 June 2020)

\begin{abstract}
We perform dynamic Monte Carlo simulations to investigate the equilibrium dynamics of hard board-like colloidal particles in oblate and prolate nematic liquid crystals. In particular, we characterize the particles' diffusion along the nematic director and perpendicularly to it, and observe a structural relaxation decay that strongly depends on the particle anisotropy. To assess the Gaussianity of their dynamics and eventual occurrence of collective motion, we calculate two- and four-point correlation functions that incorporate the instantaneous values of the diffusion coefficients parallel and perpendicular to the nematic director. Our simulation results highlight the occurrence of Fickian and Gaussian dynamics at short and long times, locate the minimum diffusivity at the self-dual shape, the particle geometry that would preferentially stabilise biaxial nematics, and exclude the existence of dynamically correlated particles.
\end{abstract}

DOI: 10.1103/PhysRevE.101.052702

\section{INTRODUCTION}

Colloids are systems of macromolecules or nanoparticles dispersed through a continuous medium. If the dispersed phase consists of solid particles and the continuous phase is a liquid, like an ink or a paint, then they are referred to as colloidal suspensions. The particles' ability of remaining suspended in the liquid stems from a thermal energy that must overcome the gravitational potential energy, which, by contrast, promotes sedimentation. Colloidal suspensions of anisotropic particles are complex fluids that display a particularly rich phase behavior, with liquid crystals (LCs) forming between the isotropic and the crystal phase. LCs are states of matter with intermediate features between those of a simple, disordered liquid and those of a crystalline solid. More specifically, they flow like liquids, but their building blocks are able to orient along one or more spatial directions, thus closely reproducing the internal ordering of molecular crystals and their optical properties.

A renewed interest has been recently devoted to colloidal suspensions of anisotropic particles with a biaxial geometry, such as board-like and bent-core particles, whose orientation in space is fully identified by two independent orthogonal axes. Biaxial particles are, in principle, excellent candidates to form biaxial nematic $\left(\mathrm{N}_{\mathrm{B}}\right) \mathrm{LCs}$, whose existence, predicted by a generalized Maier-Saupe theory proposed by Freiser exactly half a century ago [1,2], is still the object of a fervent research interest among experimental, computational and theoretical

\footnotetext{
*acuemen@upo.es

†alessandro.patti@manchester.ac.uk
}

Published by the American Physical Society under the terms of the Creative Commons Attribution 4.0 International license. Further distribution of this work must maintain attribution to the author(s) and the published article's title, journal citation, and DOI. groups [3-16]. This interest is rooted in the captivating idea of manufacturing biaxial LCDs, by many envisaged as the nextgeneration fast display technology, but still at an embryonic stage due to the difficulty of unambiguously identifying stable molecular $\mathrm{N}_{B}$ phases. By contrast, stable colloidal $\mathrm{N}_{B}$ phases have been observed for the first time approximately ten years ago in systems of polydisperse prolate goethite particles [3].

Despite such an intense research activity aiming at fully unveiling the phase and aggregation behavior of biaxial particles [17], much less attention has been devoted to their dynamics. On one hand, this is not surprising as, apart from the especially successful case of the Gay-Berne fluid [18] and the purely orientational pair potential proposed by Straley [19], most of the model particles with a biaxial geometry lack a suitable interaction potential to compute time trajectories and are often modelled as arrays of spherical beads [20]. On the other hand, it is not an easy task to predict how and, especially, over what time scales the $\mathrm{N}_{B}$ phase would respond to external stimuli and hence quantify the advantages of employing them in LCDs, if an insight into their dynamical behavior is missing. For instance, it is relevant to know how the shape anisotropy of biaxial particles determines their ability of diffusing in a crowded and ordered mesophase, such as a nematic LC; it is also important to know whether these particles are dynamically correlated as this would influence the response time of the whole phase to applied forces. In this context, the dynamic Monte Carlo (DMC) simulation method plays a very important role as it provides a theoretical framework to mimic the dynamics of colloidal particles of any possible shape without integrating stochastic or deterministic differential equations as done, respectively, in Brownian dynamics (BD) or molecular dynamics (MD) simulations [21-23]. The DMC method, which shows excellent quantitative agreement with BD simulations and can be between 10 and 20 times faster than BD [23], is especially useful to mimic the dynamics of hard-core particles, which cannot be straightaway reproduced by $\mathrm{MD}$ or BD simulations. Consequently, the dynamics of 


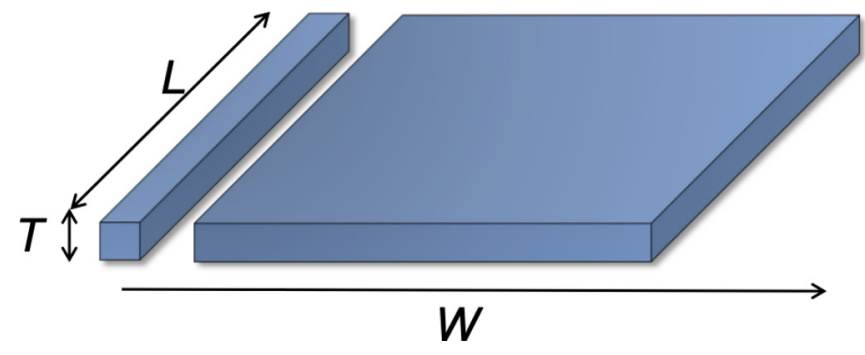

FIG. 1. Model HBPs with thickness $T$, length $L=12 T$, and width $W$. The particle width is a simulation parameter with values between $W=T$ (rod-like particles) and $W=L$ (disk-like particles).

biaxial particles interacting via a mere steric repulsion can be analysed by DMC simulations. The main goal of the present work is therefore investigating the equilibrium dynamics of prolate and oblate hard board-like particles in nematic LCs and characterize their structural relaxation decay.

This paper is organized as follows. In Sec. II, we introduce the model and simulation methodology, referring the reader to our previous contributions for specific details on the implementation of the DMC technique. The relevant correlation functions to assess the dynamics are also discussed in this section. In Sec. III, we discuss the main features of the equilibrium dynamics of HBPs and, finally, in Sec. IV, we draw our conclusions.

\section{MODEL AND SIMULATIONS}

The colloidal particles studied in this work are modelled as hard board-like particles (HBPs), whose main geometrical features are shown in Fig. 1. They are rectangular prisms of thickness $T$, length $L=12 T$, and width $T \leqslant W \leqslant L$. The particle thickness, $T$, is our unit length and is kept constant, while the particle width, $W$, is a simulation parameter that varies between $T$ and $L$ in order to reproduce prolate (rod-like) and oblate (disk-like) particles, respectively. At $W=\sqrt{L T}$, HBPs are not oblate nor prolate, but display an ambivalent nature that can produce nematic LCs with oblate, prolate or biaxial symmetry. This special geometry is referred to as self-dual shape.

The width-to-thickness ratio, $W^{*} \equiv W / T$, and the system packing fraction, $\eta \equiv N_{p} v_{0} / V$, completely determine the phase behavior and dynamics of these systems. In the latter definition, $V$ is the volume of the simulation box, $N_{p}=2000$ the number of HBPs, and $v_{0}=12 W T^{2}$ the particle volume. The phase diagram of monodisperse HBPs, studied by Monte Carlo (MC) simulations, reveals the existence of oblate and prolate nematic and columnar liquid-crystalline phases as well as biaxial smectics [13]. More recently, simulations of especially large systems suggested that the prolate columnar phase might actually be metastable with respect to either a smectic phase or a crystal phase, depending on the system density [24]. Over the last ten years, the quest for the elusive biaxial nematic $\left(\mathrm{N}_{\mathrm{B}}\right)$ phase has produced a number of theoretical [4], simulation $[10,14,15]$ and experimental [8] studies that confirm the difficulty of obtaining a stable $\mathrm{N}_{B}$ phase of HBPs. The focus of the present contribution is investigating and characterizing the relaxation dynamics of prolate $\left(\mathrm{N}^{+}\right)$and oblate $\left(\mathrm{N}^{-}\right)$uniaxial nematic phases of HBPs. To this end, we first performed standard MC simulations in the canonical ensemble to equilibrate the systems at the packing fraction, $\eta=0.34$, at which $\mathrm{N}^{+}$and $\mathrm{N}^{-}$phases are known to be stable over the full set of aspect ratios studied here, namely $1 \leqslant$ $W^{*} \leqslant \sqrt{L^{*}} \approx 3.46$ for $\mathrm{N}^{+}$and $3.46 \leqslant W^{*} \leqslant 12$ for $\mathrm{N}^{-}[13]$, where $L^{*} \equiv L / T$. To study the relaxation dynamics of these systems, we performed dynamic Monte Carlo (DMC) simulations in the canonical ensemble. Details of the DMC method are available to the interested reader in our former works [21-23]. Here, we briefly summarize the main points that are essential to gain a general insight.

DMC simulations are performed in cubic boxes with periodic boundaries and, since our intention is to mimic the time evolution of physical systems, no unphysical moves, such as cluster moves or particle swaps, are allowed. One DMC cycle consists of $N_{p}$ attempts of simultaneously displacing and rotating randomly selected HBPs. These combined moves are accepted or rejected according to the standard Metropolis algorithm, that is with probability $\min [1, \exp (-\beta \Delta E)]$, where $\Delta E$ is the energy difference resulting from the particle's move and $\beta$ the inverse temperature. Because the interactions between HBPs are modelled by a hard-core potential, moves are always accepted unless an overlap is produced. Overlap tests are based on the separating axes algorithm proposed by Gottschalk et al. [25] and later adapted by John and Escobedo to investigate the phase behavior of colloidal HBPs with square cross section [26]. The position of the center of mass of a given particle $j$ is updated by decoupling the displacement into three contributions: $\delta \mathbf{r}_{j}=X_{T} \hat{\mathbf{u}}_{j}+X_{W} \hat{\mathbf{v}}_{j}+X_{L} \hat{\mathbf{w}}_{j}$, where $\hat{\mathbf{u}}_{j}, \hat{\mathbf{v}}_{j}$, and $\hat{\mathbf{w}}_{j}$ are unit vectors parallel to the particle thickness, width and length, respectively. The magnitude of displacements along the three particle axes is selected within uniform distributions that depend on the particle translational diffusivities at infinite dilution, $D_{\alpha, j}^{t r a}$, with $\alpha=T, W$, or $L$. In particular,

$$
\left|X_{\alpha}\right| \leqslant \sqrt{2 D_{\alpha, j}^{t r a} \delta t_{\mathrm{MC}}}
$$

where $\delta t_{\mathrm{MC}}$ is the timescale for a single DMC cycle. As far as the rotations are concerned, we attempt to reorient the particle's axes $\hat{\mathbf{w}}_{j}, \hat{\mathbf{v}}_{j}$, and $\hat{\mathbf{u}}_{j}$ by three consecutive rigid rotations around $L, W$, and $T$, respectively. These rotations must satisfy the following condition:

$$
\left|Y_{\alpha}\right| \leqslant \sqrt{2 D_{\alpha, j}^{r o t} \delta t_{\mathrm{MC}}}
$$

where $D_{\alpha, j}^{r o t}$ are the particle rotational diffusivities at infinite dilution. The diffusion coefficients $D_{\alpha}^{t r a}$ and $D_{\alpha}^{r o t}$ have been estimated by employing $\mathrm{HYDRO}^{++}$, a program that calculates the solution properties of macromolecules and colloidal particles by approximating their shape and volume with an array of spherical beads of arbitrary size [27,28]. Numerical values of the translational and rotational diffusion coefficients used in this work are given in Table I in units of $D_{0} \equiv T^{2} \tau^{-1}$, where $\tau$ is the time unit. In order to compare the dynamics of the systems listed in Table I, it is crucial to recover the actual timescale of the Brownian dynamics (BD), $\delta t_{\mathrm{BD}}$ [21-23]. To this end, one needs to rescale the DMC time scale, which has been set to $\delta t_{\mathrm{MC}}=10^{-2} \tau$ for all the systems studied, via the 
TABLE I. Infinite-dilution translational and rotational diffusion coefficients of HBPs with different width-to-thickness ratios, $W^{*}$, as obtained from $\mathrm{HYDRO}^{++}[27,28]$.

\begin{tabular}{lccccc}
\hline \hline$W^{*}$ & $D_{T}^{\text {tra }} / D_{0}$ & $D_{W}^{\text {tra }} / D_{0}$ & $D_{L}^{\text {tra }} / D_{0}$ & $D_{T}^{\text {rot }} / D_{0}$ & $D_{W}^{\text {rot }} / D_{0}$ \\
\hline 1 & $2.2 \times 10^{-2}$ & $2.2 \times 10^{-2}$ & $3.1 \times 10^{-2}$ & $1.1 \times 10^{-3}$ & $1.1 \times 10^{-3}$ \\
2.5 & $1.7 \times 10^{-2}$ & $1.9 \times 10^{-2}$ & $2.4 \times 10^{-3}$ & $7.9 \times 10^{-4}$ & $7.1 \times 10^{-4}$ \\
3 & $1.6 \times 10^{-2}$ & $1.8 \times 10^{-2}$ & $2.2 \times 10^{-2}$ & $7.3 \times 10^{-4}$ & $6.5 \times 10^{-4}$ \\
3.46 & $1.5 \times 10^{-2}$ & $1.8 \times 10^{-2}$ & $2.1 \times 10^{-2}$ & $6.7 \times 10^{-4}$ & $6.0 \times 10^{-4}$ \\
4 & $1.4 \times 10^{-2}$ & $1.7 \times 10^{-2}$ & $2.0 \times 10^{-2}$ & $6.2 \times 10^{-4}$ & $5.6 \times 10^{-4}$ \\
6 & $1.2 \times 10^{-2}$ & $1.5 \times 10^{-2}$ & $1.7 \times 10^{-2}$ & $4.6 \times 10^{-4}$ & $4.4 \times 10^{-4}$ \\
8 & $9.4 \times 10^{-3}$ & $1.4 \times 10^{-2}$ & $1.5 \times 10^{-2}$ & $3.5 \times 10^{-4}$ & $3.6 \times 10^{-4}$ \\
12 & $6.5 \times 10^{-3}$ & $1.2 \times 10^{-2}$ & $1.2 \times 10^{-2}$ & $2.1 \times 10^{-4}$ & $2.6 \times 10^{-4}$ \\
\hline \hline
\end{tabular}

acceptance rate $\mathcal{A}$ :

$$
\delta t_{\mathrm{BD}}=\frac{\mathcal{A}}{3} \delta t_{\mathrm{MC}}
$$

This result has been applied to rescale the dynamical properties that characterize the structural relaxation of our systems, namely (i) the mean-square displacement; (ii) the self-part of the van Hove function; (iii) the self-intermediate scattering function; and (iv) the dynamic susceptibility.

The mean square displacement (MSD) is the ensemble average of the particles' displacement from their position over a given time window and is defined as

$$
\left\langle\Delta r^{2}(t)\right\rangle=\frac{1}{N_{p}}\left\langle\sum_{j=1}^{N_{p}}\left(\mathbf{r}_{j}(t)-\mathbf{r}_{j}(0)\right)^{2}\right\rangle,
$$

where $\mathbf{r}_{j}$ is the position vector of particle $j$ and the angular brackets indicate ensemble average over all the HBPs and at least 100 independent trajectories. The self-part of the van Hove function (s-VHF) provides the probability distribution of the HBPs' displacements at time $t_{0}+t$, given their position at time $t_{0}$ and reads

$$
G_{s}(r, t)=\frac{1}{N_{p}}\left\langle\sum_{j=1}^{N_{p}} \delta\left(r-\left|\mathbf{r}_{j}\left(t_{0}+t\right)-\mathbf{r}_{j}\left(t_{0}\right)\right|\right)\right\rangle,
$$

where the symbol $\delta$ is the Dirac delta. If the distribution of the displacements over time is Gaussian, then $G_{s}(r, t)$ can be approximated by a Gaussian function that reads

$$
G_{s, d}^{\prime}(r, t)=\left(4 \pi D_{t} t\right)^{-d / 2} \exp \left(-\frac{r^{2}}{4 D_{t} t}\right),
$$

where $D_{t}$ is the instantaneous diffusion coefficient and $d$ the dimensionality of the system dynamics. In particular, $G_{s, 1}^{\prime}$ and $G_{s, 2}^{\prime}$ are the Gaussian approximations of the parallel and perpendicular s-VHFs. As far as $G_{s, 3}^{\prime}$ is concerned, we have recently shown that it would not correctly determine the Gaussianity of the displacements in anisotropic systems, and thus proposed a Gaussian distribution that has an ellipsoidal, rather than spherical, symmetry [29]. More specifically, for prolate particles, the Gaussian approximation of the s-VHF function takes the following form:

$$
G_{s, 3}^{\prime}(r, t)=\frac{\Omega}{(4 \pi t)^{3 / 2}} \exp \left(-\frac{r^{2} t^{-1}}{4 D_{t, \|}}\right) \frac{F\left(r \Delta_{p}^{1 / 2}\right)}{r \Delta_{p}^{1 / 2}}
$$

and for oblate particles

$$
G_{s, 3}^{\prime}(r, t)=\frac{\Omega \sqrt{\pi}}{2(4 \pi t)^{3 / 2}} \exp \left(-\frac{r^{2} t^{-1}}{4 D_{t, \perp}}\right) \frac{\operatorname{erf}\left(r \Delta_{o}^{1 / 2}\right)}{r \Delta_{o}^{1 / 2}}
$$

where $D_{t, \|}$ and $D_{t, \perp}$ are, respectively, the instantaneous values of the diffusion coefficients in the direction parallel and perpendicular to $\hat{\mathbf{n}}$, respectively, $F(\cdots)$ is the Dawson's integral, $\operatorname{erf}(\cdots)$ the error function, $\Omega=1 /\left(D_{t, \perp}^{2} D_{t, \|}\right)^{1 / 2}$, and $\Delta_{p}=$ $-\Delta_{o}=1 /\left(4 D_{t, \perp} t\right)-1 /\left(4 D_{t, \|} t\right)$.

The self-intermediate scattering function (s-ISF) is useful to gain an insight into the timescales associated to the system's structural relaxation occurring over a length $2 \pi /|\mathbf{k}|$, where $\mathbf{k}$ is a wave vector that corresponds to the location of the relevant peaks in the static structure factor. It is defined as

$$
F_{s}(k, t)=\frac{1}{N_{p}}\left\langle\sum_{j=1}^{N_{p}} \exp \left[i \mathbf{k} \cdot\left(\mathbf{r}_{j}\left(t+t_{0}\right)-\mathbf{r}_{j}\left(t_{0}\right)\right)\right]\right\rangle .
$$

The fluctuations of the self-intermediate scattering function define the so-called dynamical susceptibility, $\chi_{4}(k, t)$, which provides useful information on the extent of dynamic heterogeneities or, equivalently, on the number of dynamically correlated particles:

$$
\chi_{4}(k, t)=N_{p}\left[\left\langle f_{s}^{2}(k, t)\right\rangle-F_{s}^{2}(k, t)\right],
$$

where $f_{s}(k, t)=\sum_{j=1}^{N_{p}} \cos \left(\mathbf{k}\left[\left(\mathbf{r}_{j}\left(t+t_{0}\right)-\mathbf{r}_{j}\left(t_{0}\right)\right)\right] / N_{p}\right.$ is the real part of the instantaneous value of the s-ISF.

\section{RESULTS}

In this section, we analyze the main features of the equilibrium dynamics of HBPs in $\mathrm{N}^{-}$and $\mathrm{N}^{+}$phases by DMC simulations. All the results shown here have been rescaled with the acceptance rate to recover the time scale of the Brownian dynamics [21-23]. We stress that this is an essential step in order to correctly compare the dynamic properties of different systems. The typical behavior detected in dense colloidal LCs generally consists of a short-time diffusive regime, where the MSD depends linearly on time, followed by an intermediate regime where the particles' motion is slowed down by the surrounding neighbours, referred to as caging effect, and a long-time diffusion regime controlled by the collisions between particles [30-39]. The intermediate regime becomes 


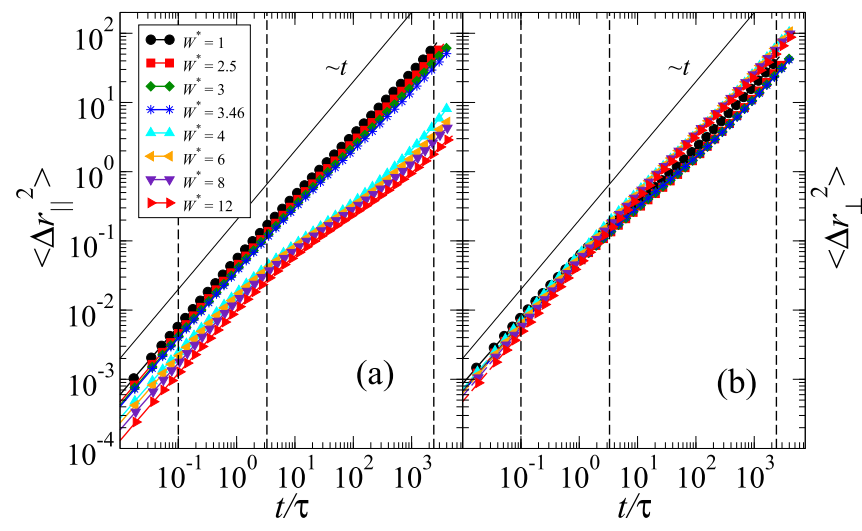

FIG. 2. Mean square displacement of oblate and prolate HBPs with width-to-thickness ratio indicated in the legend. Frames (a) and (b) refer to the directions parallel and perpendicular to the nematic director, respectively. The vertical dashed lines correspond to $t / \tau=$ $0.1,3.3$, and 2400. The solid lines highlight the linear dependence of the MSD on time at short and long timescales.

more and more relevant at increasing densities, with the MSD developing a plateau that can extend several time decades in especially dense colloidal suspensions, such as columnar LCs [31]. Nematic LCs of uniaxial particles usually show a very short, almost evanescent, intermediate regime, especially at densities very close to the isotropic-nematic phase transition. Similar tendencies are also noticed here in $\mathrm{N}^{-}$and $\mathrm{N}^{+}$phases of biaxial HBPs. In particular, the MSD in the direction of $\hat{\mathbf{n}}$, shown in Fig. 2(a), reveals a relatively smooth transition from short-time to long-time diffusion in systems of prolate geometries $\left(1 \leqslant W^{*} \leqslant 3.46\right)$. Oblate HBPs show a slightly different behavior that suggests a more pronounced caging effect as compared to that of their prolate counterparts. In this case, the inset of the long-time diffusive regime, where the effect of caging vanishes completely and $\left\langle\Delta r_{\|}^{2}\right\rangle$ recovers its linearity with time, occurs between $t / \tau \approx 2 \times 10^{3}$ and $3 \times 10^{3}$, depending on the particle's anisotropy.

By contrast, the perpendicular MSD, $\left\langle\Delta r_{\perp}^{2}\right\rangle$, reported in Fig. 2(b), is not especially influenced by the particle anisotropy, although it can be observed that, at long timescales, the MSD of oblate HBPs is slightly larger than that of prolate HBPs. To clarify the role of the shape anisotropy on the particles' ability of diffusing in nematic LCs, we have calculated the translational diffusion coefficients as $D=$ $\lim _{t \rightarrow \infty}\left\langle\Delta r^{2}\right\rangle / 2 d t$, where $d=1,2$, or 3 for parallel, perpendicular, or total MSD, respectively. The resulting diffusivities are reported in the top frame of Fig. 3 as a function of $W^{*}$. It is interesting to notice that at the prolate-oblate crossover, indicated by the vertical dashed line $W^{*}=\sqrt{L^{*}} \approx 3.46$, the parallel diffusivity, $D_{\|}$, drops abruptly by a factor of 7 , from approximately $6.5 \times 10^{-3} D_{0}$ at $W^{*}=3.46$ to $10^{-3} D_{0}$ at $W^{*}=4$. An opposite tendency is detected in the perpendicular diffusivity, $D_{\perp}$, which increases from $2.5 \times 10^{-3} D_{0}$ at $W^{*}=3.46$ to $7 \times 10^{-3} D_{0}$ at $W^{*}=4$. The combined effect of parallel and perpendicular diffusion results in a total diffusion coefficient, $D_{T O T}=\left(2 D_{\perp}+D_{\|}\right) / 3$ in Fig. 3(a), that displays a minimum exactly at the self-dual shape. Despite being the geometry that would more easily stabilise the biaxial nematic

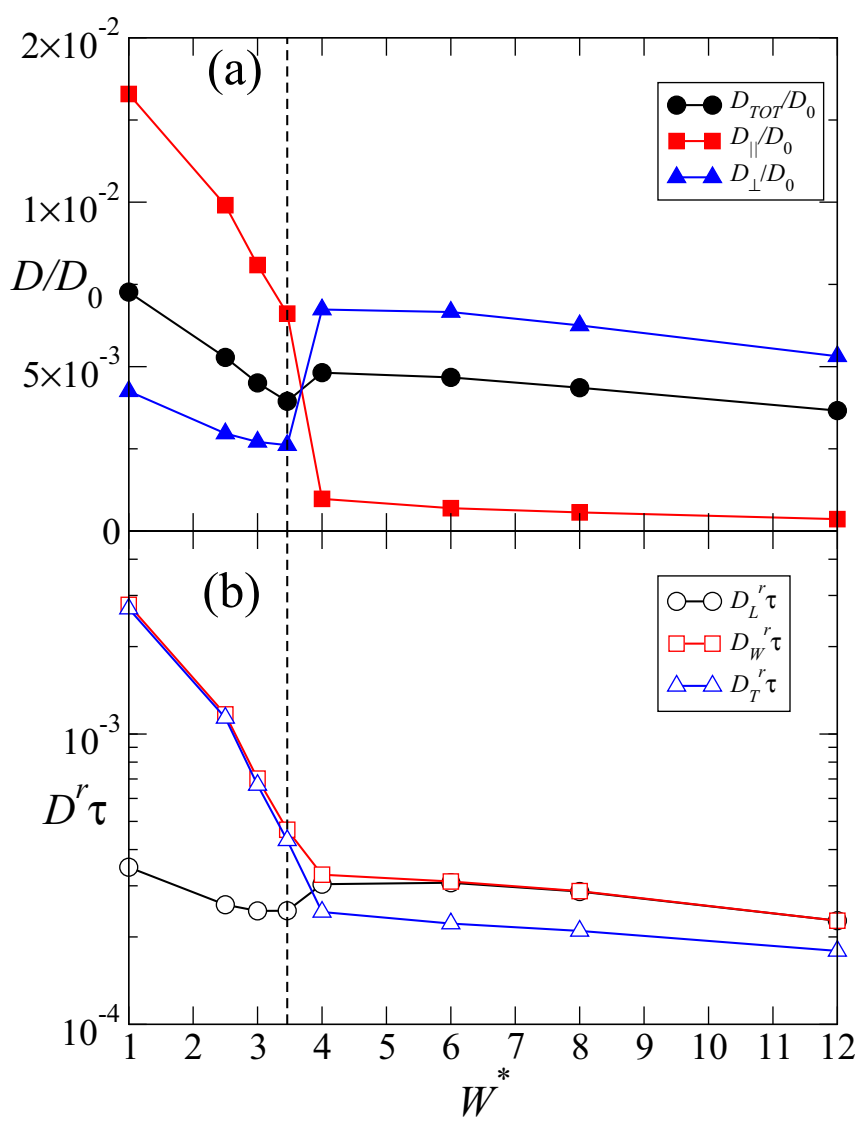

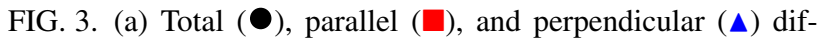
fusion coefficients of oblate and prolate HBPs in nematic LCs. (b) Rotational diffusion coefficients corresponding to the rotation of the axis parallel to $L(\bigcirc), W(\square)$, and $T(\triangle)$. The vertical dashed line at $W^{*}=\sqrt{L^{*}} \approx 3.46$ indicates the transition from prolate to oblate particle shapes. The solid lines are a guide for the eyes.

phase, the self-dual shape delays diffusion, at least for the range of particle anisotropies explored in this work.

To gain an insight into the particle orientational relaxation, we have also calculated the rotational diffusion coefficients, which are reported in Fig. 3(b). Given the biaxial geometry of the particles, there are three independent rotational diffusion coefficients, corresponding to the rotation of the three particle axes $\hat{\mathbf{w}}, \hat{\mathbf{v}}$, and $\hat{\mathbf{u}}$. These coefficients have been calculated via the orientational time-correlation functions that read [40,41]

$$
C_{\alpha}=\left\langle P_{1}[\hat{\mathbf{e}}(t) \cdot \hat{\mathbf{e}}(0)]\right\rangle
$$

with $\alpha=T, W$, or $L$ and $\hat{\mathbf{e}}=\hat{\mathbf{u}}, \hat{\mathbf{v}}$, or $\hat{\mathbf{w}}$, respectively. $P_{1}$ is the first Legendre polynomial, and the brackets indicate ensemble averages over particles and time origins. For each of the orientations, the corresponding relaxation time have been calculated as follows:

$$
\tau_{\alpha}=\int_{0}^{\infty} C_{\alpha} d t
$$

and the three different long time rotational diffusion coefficients are given by $D_{\alpha}^{r}=\tau_{\alpha} / 2$. One would expect the rotation of the axis aligned with the nematic director to be more 


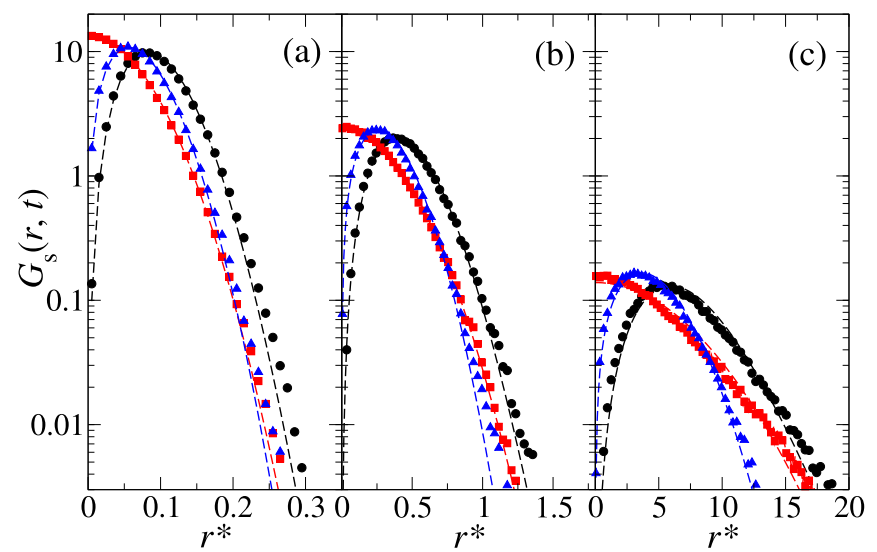

FIG. 4. Total (๑), parallel ( $\square$ ), and perpendicular $(\boldsymbol{\Delta})$ self-part of the van Hove correlation function of prolate HBPs with $W^{*}=1$. (a), (b), and (c) refer, respectively, to $t / \tau=0.1,3.3$, and 2400. Dashed lines are Gaussian distributions obtained from Eqs. (6) and (7).

difficult than those around it and thus to take longer. This is indeed observed in $\mathrm{N}^{+}$phases $\left(W^{*}<3.46\right)$, where the rotational diffusivity $D_{L}^{r}$, corresponding to the reorientation of the unit vector parallel to $L$, is about one order of magnitude smaller than $D_{W}^{r}$ and $D_{T}^{r}$. By contrast, in $\mathrm{N}^{-}$phases $\left(W^{*}>\right.$ 3.46) this difference is less significant, suggesting a relatively stronger coupling of the rotational dynamics.

The effect of shape anisotropy is not limited to the single particle ability to diffuse, but extends to the whole system by contributing to determine the degree of Gaussianity of its dynamics as well as the decay of its structural relaxation. More specifically, eventual deviations from Gaussian dynamics are estimated by computing the self-van Hove correlation functions, while the long-time relaxation of the system's density fluctuations is assessed via the self-intermediate scattering function. The self-van Hove correlation functions for nematic LCs of HBPs with $W^{*}=1$ and 12 are shown, respectively, in Figs. 4 and 5. Each frame in the two figures portrays the total (circles), parallel (squares), and perpendicular (triangles) $\mathrm{s}-\mathrm{VHF}$ at a specific time: $t / \tau=0.1$ (left frame)

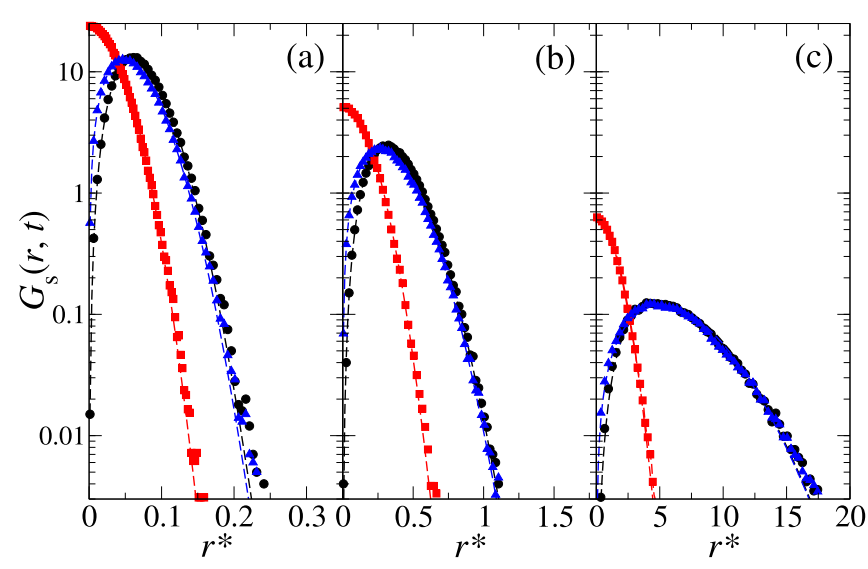

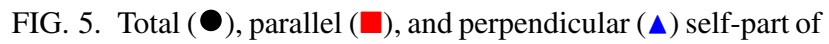
the van Hove correlation function of oblate HBPs with $W^{*}=12$. (a), (b), and (c) refer, respectively, to $t / \tau=0.1,3.3$, and 2400. Dashed lines are Gaussian distributions obtained from Eqs. (6) and (8). represents a generic instant within the short-time diffusion; $t / \tau=3.3$ (middle frame) approximately indicates the crossover from the short-time diffusion to the long-time diffusion, and matches the beginning of the caging effect for the parallel diffusion of oblate HBPs; finally, $t / \tau=2400$ (right frame) is a representative time within the fully developed long-time diffusive regime.

The Gaussianity of the parallel and perpendicular s-VHFs has been estimated by comparing the simulation results with the Gaussian distribution of Eq. (6), with $d=1$ and 2, respectively. By contrast, the Gaussianity of the total s-VHFs has been assessed with Eqs. (7) (prolate HBPs) and (8) (oblate HBPs). The agreement between DMC simulations and theory is excellent, with almost insignificant deviations from a pure Gaussian distribution of displacements detected in systems of prolate HBPs. These tiny deviations, predominantly observed at long distances, suggest the existence of a number of fast particles that are able to displace longer distances than those normally expected. By contrast, at short distances, no significant deviations between simulation and theory are noticed, thus indicating an unambiguous Gaussian behavior and the absence of particularly slow particles. The s-VHFs of HBPs with $1<W^{*}<12$ (not shown here) display a similar Gaussian behavior, the main differences being the shift of the peak towards larger distances and the gradually lower probability of observing fast particles at increasing $W^{*}$. It should also be noticed that the essentially Gaussian nature of the s-VHFs excludes, at both short and long times, where the MSD is a linear function of time, the occurrence of Fickian yet non-Gaussian (FNG) dynamics, as we had already observed in nematics of uniaxial particles [29]. This finding further confirms that not all soft-matter systems, now also including complex fluids of biaxial particles, necessarily exhibit FNG dynamics.

In the light of these considerations, we now discuss the decay of the density fluctuations in the $\mathrm{N}^{+}$and $\mathrm{N}^{-}$phases by examining the self-intermediate scattering functions, $F_{s}(k, t)$, shown in Fig. 6. The s-ISFs of oblate and prolate HBPs have been calculated by randomly selecting $5 \times 10^{4}$ wave vectors $\mathbf{k}=\left(2 \pi i_{1} / l, 2 \pi i_{2} / l, 2 \pi i_{3} / l\right)$ with $i_{1}, i_{2}$, and $i_{3}$ integer numbers and $l$ the side of the cubic simulation box, whose magnitude is $|\mathbf{k}|=2 \pi / T$. All curves in Fig. 6 display a single-step decay, which can be closely reproduced by a stretched exponential function of the type $\left.\exp \left[-\left(t / t_{r}\right)^{\alpha}\right)\right]$, with $t_{r}$ the relaxation time of the system's structural decay and $\alpha$ the stretching exponent, which ranges between 0.82 at $W^{*}=1$ and 0.88 at $W^{*}=12$. The former corresponds to the time the s-ISF takes to decays to $e^{-1}$ and it is reported in the inset of Fig. 6 as a function of the particle reduced width. The latter provides an insight into the nature of the long-time relaxation dynamics. In particular, $\alpha<1$ would indicate the emergence of separated domains of particles with different relaxation dynamics, usually referred to as dynamic heterogeneity. This behavior, often detected in supercooled liquids, glasses and gels [42-44], it is generally attributed to particles that either (i) relax exponentially at different time rates or (ii) relax nonexponentially at the same time rates $[45,46]$.

To assess the origin of dynamic heterogeneity in nematic LCs of HBPs, we calculated the dynamic susceptibility, $\chi_{4}(k, t)$, which is proportional to the number of dynamically 


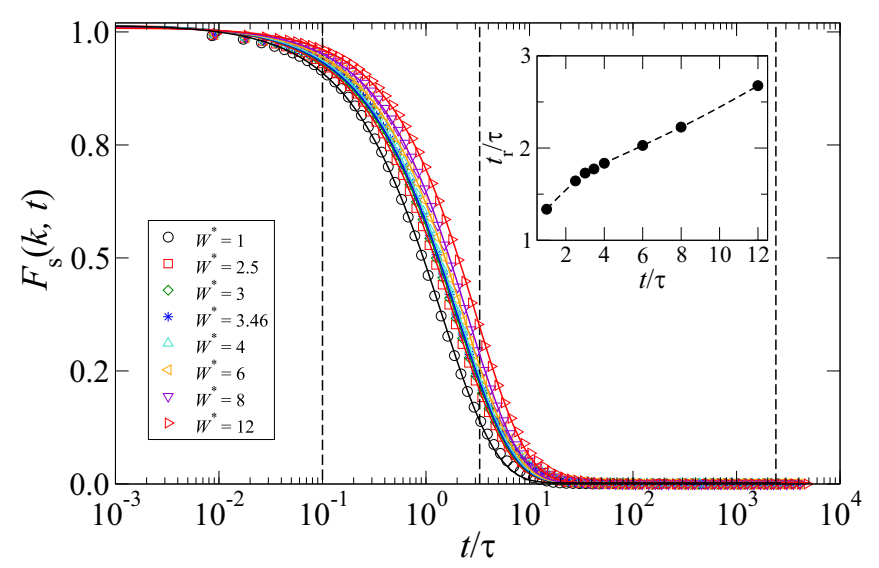

FIG. 6. Self-intermediate scattering functions calculated at $|\mathbf{k}|=$ $2 \pi / T$ for oblate and prolate HBPs, respectively. The particle widthto-thickness ratio is reported in the legend. The solid lines are stretched exponential fits of the form $\left.\exp \left[-\left(t / t_{r}\right)^{\alpha}\right)\right]$, with $t_{r}$ the relaxation time and $\alpha$ the stretching exponential (see text for details). The inset shows the relaxation time as a function of the particle anisotropy at $|\mathbf{k}|=2 \pi / T$. The solid lines are guides for the eye and the vertical dashed lines correspond to $t / \tau=0.1,3.3$, and 2400 .

correlated particles over a time $t$ and a distance $\approx 2 \pi / k$. This function hence ponders the presence of clusters that, if existed, would be formed by particles relaxing at the same time rate, thus corroborating scenario (ii). The dynamic susceptibility of $\mathrm{N}^{+}$and $\mathrm{N}^{-}$phases of HBPs, reported in Fig. 7, shows a peak at $t / \tau \approx 3.3$, at the crossover from short-time to long-time diffusive regime. The magnitude of this peak is not significant and, as expected by the relatively large value of the stretching exponent $\alpha$, which is not much lower than 1 , excludes the occurrence of HBPs that are dynamically correlated. In other words, in agreement with uniaxial disks and rods [39], there is no evidence of clusters of particles moving collectively and consequently the structural relaxation of both oblate and

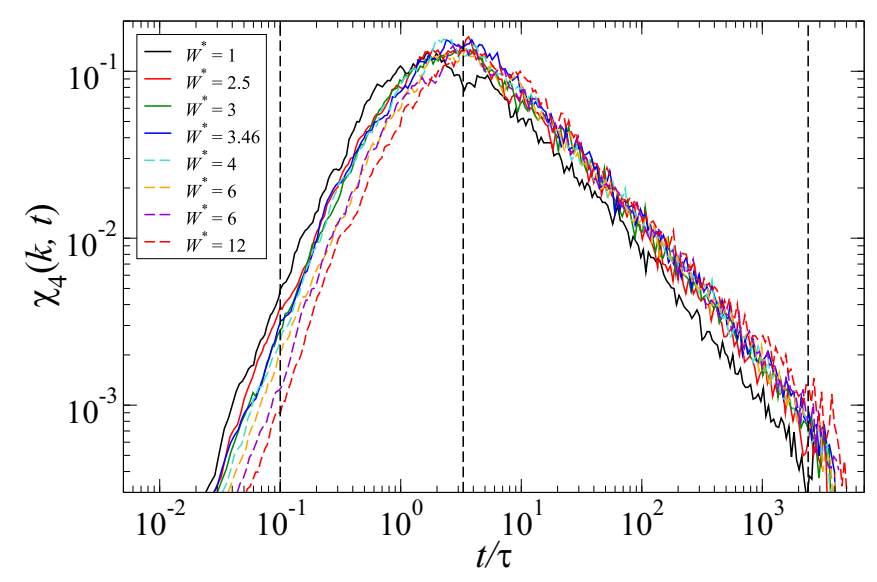

FIG. 7. Dynamic susceptibility in $\mathrm{N}^{+}$and $\mathrm{N}^{-}$LCs of HBPs at $|\mathbf{k}|=2 \pi / T$. The particle width-to-thickness ratio is reported in the legend. The vertical dashed lines correspond to $t / \tau=0.1,3.3$, and 2400 . prolate nematics of HBPs is entirely determined by the singleparticle dynamics.

\section{CONCLUSIONS}

In summary, we have studied the equilibrium dynamics of prolate and oblate HBPs in nematic LCs. To this end, we have employed the DMC simulation technique, which can accurately reproduce the Brownian dynamics of colloids. In particular, we find Fickian and Gaussian dynamics at both short-time and long-time scales, which are separated by a very smooth crossover indicating an almost completely negligible caging effect. The HBPs' anisotropy (precisely, their width-to-thickness ratio) plays a relevant role in the particle's ability to diffuse along the nematic director, $\hat{\mathbf{n}}$, and perpendicularly to it. Prolate and oblate HBPs diffuse faster in the direction parallel and perpendicular to $\hat{\mathbf{n}}$, respectively. Interestingly, despite being indicated as the most appropriate geometry for the formation of the elusive biaxial nematic phase, the self-dual shape reduces the particles' total diffusivity, which displays a minimum exactly at $W^{*}=\sqrt{L^{*}}$. Additionally, the dual shape marks a frontier between the behavior of prolate and oblate particles. This change is reflected in the different nematic symmetry, different direction of the faster component of the transnational diffusion, and for a qualitative change in the relative magnitude of the rotational diffusivities. The analysis of the self-van Hove correlation functions reveals the full Gaussianity of the distribution of displacements over time. Very small deviations are actually observed in $\mathrm{N}^{+}$phases at large distances, suggesting the existence of few fast particles that are not found in $\mathrm{N}^{-}$phases. Finally, the structural relaxation decay, investigated with the selfintermediate scattering functions, exhibits a stretched exponential behavior with a relaxation time that approximately increases by a factor 2 with increasing particle width-tothickness ratio from $W^{*}=1$ to 12 . The stretched exponential nature of the structural relaxation of dense systems, such as supercooled liquids, glasses, and gels, usually suggests the occurrence of particles dynamically correlated and eventually displaying collective dynamics [42-44]. To assess the emergence of this phenomenon, we calculated the four-point dynamic susceptibility, which measures the magnitude of the dynamic correlations. However, as also noticed for uniaxial particles in nematic LCs [39], no sign of dynamic clusters and collective dynamics has been detected. Their occurrence in positionally ordered LC phases is currently under investigation.

\section{ACKNOWLEDGMENTS}

A.C. acknowledges the Spanish Ministerio de Ciencia, Innovación y Universidades and FEDER for funding (Project No. PGC2018-097151-B-I00) and C3UPO for the HPC facilities provided. A.P. acknowledges financial support from EPSRC under Grant Agreement No. EP/N02690X/1 and the Leverhulme Trust Research Project Grant No. RPG-2018-415. 
[1] W. Maier and A. Saupe, Eine einfache molekular-statistische Theorie der nematischen kristallinflssigen Phase. Teil 1, Z. Naturforsch. 14, 882 (1959).

[2] M. J. Freiser, Ordered States of a Nematic Liquid, Phys. Rev. Lett. 24, 1041 (1970).

[3] E. van den Pol, A. V. Petukhov, D. M. E. Thies-Weesie, D. V. Byelov, and G. J. Vroege, Experimental Realization of Biaxial Liquid Crystal Phases in Colloidal Dispersions of Boardlike Particles, Phys. Rev. Lett. 103, 258301 (2009).

[4] S. Belli, A. Patti, M. Dijkstra, and R. van Roij, Polydispersity Stabilizes Biaxial Nematic Liquid Crystals, Phys. Rev. Lett. 107, 148303 (2011).

[5] S. D. Peroukidis, A. G. Vanakaras, and D. J. Photinos, Supramolecular nature of the nematic-nematic phase transitions of hard boardlike molecules, Phys. Rev. E. 88, 062508 (2013).

[6] A. B. G. M. Leferink op Reinink, S. Belli, R. van Roij, M. Dijkstra, A. V. Petukhov, and G. J. Vroege, Tuning biaxiality of nematic phases of board-like colloids by an external magnetic field, Soft Matter 10, 446 (2014).

[7] Y. Martínez-Ratón, M. González-Pinto, and E. Velasco, Biaxial nematic phase stability and demixing behavior in monolayers of rod-plate mixtures, Phys. Chem. Chem. Phys. 18, 24569 (2016).

[8] Y. Yang, G. Chen, S. Thanneeru, J. He, K. Liu and Z. Nie, Synthesis and assembly of colloidal cuboids with tunable shape biaxiality, Nat. Commun. 9, 4513 (2018).

[9] H. Mundoor, S. Park, B. Senyuk, H. H. Wensink, I. I. Smalyukh, Hybrid molecular-colloidal liquid crystals, Science 360, 768 (2018).

[10] S. Dussi, S. Tasios, T. Drwenski, R. van Roij and M. Dijkstra, Hard Competition: Stabilizing the Elusive Biaxial Nematic Phase in Suspensions of Colloidal Particles with Extreme Lengths, Phys. Rev. Lett. 120, 177801 (2018).

[11] S. Orlandi, L. Muccioli, R. Berardi, From rod-like to disc-like Gay-Berne biaxial nematics and back, Liq. Crys. 45, 2400 (2018).

[12] T. Drwenski, R. van Roij, The effect of flexibility and bend angle on the phase diagram of hard colloidal boomerangs, Mol. Phys. 116, 2812 (2018).

[13] A. Cuetos, M. Dennison, A. Masters, and A. Patti, Phase behavior of hard board-like particles, Soft Matter 13, 4720 (2017).

[14] A. Patti and A. Cuetos, Monte Carlo simulation of binary mixtures of hard colloidal cuboids, Mol. Sim. 44, 516 (2018).

[15] A. Cuetos, E. M. Rafael, D. Corbett, A. Patti, Biaxial nematics of hard cuboids in an external field, Soft Matter 19, 1922 (2019).

[16] M. Chiappini, T. Drwenski, R. van Roij, M. Dijkstra, Biaxial, Twist-Bend, and Splay-Bend Nematic Phases of BananaShaped Particles Revealed by Lifting the "Smectic Blanket," Phys. Rev. Lett. 123, 068001 (2019).

[17] Biaxial Nematic Liquid Crystals. Theory, Simulation and Experiment, edited by G. R. Luckhurst and T. J. Sluckin (Wiley, New York, 2105).

[18] J. G. Gay and B. J. Berne, Modification of the overlap potential to mimic a linear site-site potential, J. Chem. Phys. 74, 3316 (1981).

[19] J. P. Straley, Ordered phases of a liquid of biaxial particles, Phys. Rev. A 10, 1881 (1974).

[20] G. Cinacchi, A. M. Pintus, A. Tani, Diffusion of helical particles in the screw-like nematic phase, J. Chem. Phys. 145, 134903 (2016).
[21] A. Patti and A. Cuetos, Brownian dynamics and dynamic Monte Carlo simulations of isotropic and liquid crystal phases of anisotropic colloidal particles: A comparative study, Phys. Rev. E 86, 011403 (2012).

[22] A. Cuetos and A. Patti, Equivalence of Brownian dynamics and dynamic Monte Carlo simulations in multicomponent colloidal suspensions, Phys. Rev. E 92, 022302 (2015).

[23] D. Corbett, A. Cuetos, M. Dennison, and A. Patti, Dynamic Monte Carlo algorithm for out-of-equilibrium processes in colloidal dispersions, Phys. Chem. Chem. Phys. 20, 15118 (2018).

[24] S. Dussi, M. Chiappini, and M. Dijkstra, On the stability and finite-size effects of a columnar phase in single-component systems of hard-rod-like particles, Mol. Phys. 116, 2792 (2018).

[25] S. Gottschalk, M. C. Lin, and D. Manocha, OBBTree: A hierarchical structure for rapid interference detection, SIGGRAPH '96: Proceedings of the 23rd Annual Conference on Computer Graphics and Interactive Technique, Vol. 15 ( ACM, Inc, 1996), pp. 170-180.

[26] B. S. John and F. A. Escobedo, Phase behavior of colloidal hard tetragonal parallelepipeds (cuboids): A Monte Carlo simulation study, J. Phys. Chem. B 109, 23008 (2005).

[27] B. Carrasco and J. García de la Torre, Hydrodynamic properties of rigid particles. Comparison of different modeling and computational strategies, Biophysical Journal 76, 3044 (1999).

[28] J. García de la Torre, G. del Rio, and A. Ortega, Improved calculation of rotational diffusion and intrinsic viscosity of bead models for macromolecules and nanoparticles, J. Phys. Chem. B 111, 955 (2007).

[29] A. Cuetos, N. Morillo, and A. Patti, Fickian yet non-Gaussian diffusion is not ubiquitous in soft matter, Phys. Rev. E 98, 042129 (2018).

[30] M. Bier, R. van Roij, M. Dijkstra, and P. van der Schoot, SelfDiffusion of Particles in Complex Fluids: Temporary Cages and Permanent Barriers, Phys. Rev. Lett. 101, 215901 (2008).

[31] A. Patti, D. El Masri, R. van Roij, and M. Dijkstra, Stringlike Clusters and Cooperative Interlayer Permeation in Smectic Liquid Crystals Formed by Colloidal Rods, Phys. Rev. Lett. 103, 248304 (2009).

[32] G. Cinacchi and L. De Gaetani, Mechanism of diffusion in the smectic-A phase of wormlike rods studied by computer simulation, Phys. Rev. E 79, 011706 (2009).

[33] R. Matena, M. Dijkstra, and A. Patti, Non-Gaussian dynamics in smectic liquid crystals of parallel hard rods Rik, Phys. Rev. E 81, 021704 (2010).

[34] A. Patti, D. El Masri, R. van Roij, and M. Dijkstra, Collective diffusion of colloidal hard rods in smectic liquid crystals: Effect of particle anisotropy, J. Chem. Phys. 132, 224907 (2010).

[35] S. Belli, A. Patti, M. Dijkstra, and R. van Roij, Heterogeneous dynamics in columnar liquid crystals of parallel hard rods, J. Chem. Phys. 133, 154514 (2010).

[36] A. Patti, S. Belli, M. Dijkstra, and R. van Roij, Relaxation dynamics in the columnar liquid crystal phase of hard platelets, Soft Matter 7, 3533 (2011).

[37] M. Piedrahita, A. Cuetos, and B. Martínez-Haya, Transport of spherical colloids in layered phases ofbinary mixtures with rodlike particles, Soft Matter 11, 3432 (2015).

[38] S. D. Peroukidis and S. H. L. Klapp, Orientational order and translational dynamics of magnetic particle assemblies in liquid crystals, Soft Matter 12, 6841 (2016). 
[39] N. Morillo, A. Patti, and A. Cuetos, Brownian dynamics simulations of oblate and prolate colloidal particles in nematic liquid crystals, J. Chem. Phys. 150, 204905 (2019).

[40] J. P. Hansen and I. R. McDonald, Theory of Simple Liquids, 3rd ed. (Academic Press, London, 2006).

[41] D. M. Heyes, Translational and rotational diffusion of rod shaped molecules by molecular dynamics simulations, J. Chem. Phys. 150, 184503 (2019).

[42] W. Kob, C. Donati, S. J. Plimpton, P. H. Poole, and S. C. Glotzer, Dynamical Heterogeneities in a Supercooled LennardJones Liquid, Phys. Rev. Lett. 79, 2827 (1997).

[43] Z. Zheng, R. Ni, F. Wang, M. Dijkstra, Y. Wang, and Y. Han, Structural signatures of dynamic heterogeneities in monolayers of colloidal ellipsoids, Nat. Commun. 5, 3829 (2014).

[44] J. Colombo and E. Del Gado, Self-assembly and cooperative dynamics of a model colloidal gel network, Soft Matter 10, 4003 (2014).

[45] R. Richert, Homogeneous dispersion of dielectric responses in a simple glass, J. Non-Cryst. Solids 172-174, 209 (1994).

[46] M. D. Ediger, Spatially heterogeneous dynamics in supercooled liquids, Annu. Rev. Phys. Chem. 51, 99 (2000).

Correction: Table I was inadvertently deleted during the production process and has been inserted appropriately. 